Les lasers et leurs applications scientifiques et médicales

\title{
Les lasers moléculaires
}

H. Brunet

LASERDOT Groupe Aérospatiale, Route de Nozay, 91460 Marcoussis, France

\section{INTRODUCTION}

C'est au cours des années 1964-65 qu'apparurent les lasers moléculaires les plus connus, le laser à $\mathrm{CO}_{2}$ et le laser $\mathrm{HCl}$, précurseur des lasers chimiques de grande puissance. Ces lasers émettent dans le proche infrarouge, sur les transitions de vibration-rotation des molécules. De ce fait, ils présentent des propriétés et des caractéristiques différentes de celles des lasers fonctionnant sur des transitions atomiques ou ioniques. Dans le premier chapitre de l'exposé, nous présenterons un rappel des propriétés spécifiques des lasers moléculaires. Dans le deuxième chapitre, on s'intéressera aux lasers à $\mathrm{CO}_{2}$, continus, impulsionnels ou guides d'ondes. Enfin, dans le troisième et dernier chapitre, on présentera les caractéristiques des lasers chimiques, essentiellement celles des lasers HF/DF, seuls lasers chimiques ayant connu un développement intéressant.

\section{GÉNÉRALITÉS}

\subsection{Vibration et rotation}

Dans cet exposé, nous ne nous intéresserons qu'aux transitions de vibration-rotation de l'état électronique fondamental de la molécule et non aux transitions entre états électroniques qui peuvent conduire à d'autres lasers moléculaires (Brunet 1973).

Les niveaux d'énergie d'une molécule diatomique sont caractérisés par leur énergie de vibration $E_{v}=(v+1 / 2)$ hv où $v$ est le nombre quantique de vibration, $v$ la fréquence et par l'énergie de rotation $\mathrm{E}_{\mathrm{J}}=\mathrm{J}(\mathrm{J}+1)$ Bhc où $\mathrm{J}$ est le nombre quantique de rotation et $\mathrm{B}$ la constante rotationnelle. Les transitions entre niveaux de rotation respectent la règle de sélection $\Delta \mathrm{J}= \pm 1$ (branche $\mathrm{P}, \Delta \mathrm{J}=-1$ - branche $\mathrm{R}, \Delta \mathrm{J}=1$ ).

Les molécules polyatomiques à $\mathrm{N}$ atomes présentent $3 \mathrm{~N}-5$ modes de vibration et le cas particulier de la molécule $\mathrm{CO}_{2}$ sera étudié au chapitre 2 . 


\subsection{Populations rovibrationnelles}

En général, la population d'un état vibrationnel est fixée par le processus d'excitation. Par contre, sauf cas particulier, les populations des états rotationnels sont fixés par la température et suivent une distribution de Boltzmann de sorte que la population d'un niveau rotationnel est donnée par :

$$
\mathrm{n}_{\mathrm{v}, \mathrm{J}}=\mathrm{N}_{\mathrm{v}} \mathrm{f}_{\mathrm{J}} \text { où } \mathrm{f}_{\mathrm{J}}=\frac{2 \mathrm{~J}+1}{Q_{R}} \exp -\left(\frac{\mathrm{BJ}(\mathrm{J}+1) \mathrm{hc}}{\mathrm{kT}}\right)
$$

où $N_{v}$ est la population totale de l'état vibrationnel v avec $Q_{R}$ donné par :

$$
\begin{aligned}
& Q_{R}=\frac{k T}{B h c} \\
& \text { La fonction } f_{J} \text { présente un maximum pour } J_{\max }=\sqrt{\frac{k T}{2 B h c}}-\frac{1}{2} \text { (voir Herzberg, 1961). }
\end{aligned}
$$

\subsection{Temps de vie radiatif}

Contrairement au cas des transitions atomiques (ou entre niveaux électroniques), les temps de vie sont très longs (ms et plus) et ce sont les collisions qui fixent la durée de vie du niveau.

\subsection{Processus collisionnels - Echanges V-T et V-V}

Lors des collisions entre molécules, de même espèce ou non, on distingue :

- l'échange V-T :

$$
\mathrm{HF}(\mathrm{v})+\mathrm{M} \rightarrow \mathrm{HF}(\mathrm{v}-1)+\mathrm{M}+\Delta \mathrm{E}
$$

où la molécule HF perd un quantum de vibration qui est finalement transformé en chaleur.

- l'échange V-V :

$$
\mathrm{HF}(\mathrm{v})+\mathrm{AB}(0) \rightarrow \mathrm{HF}(\mathrm{v}-1)+\mathrm{AB}(1)
$$

où le quantum d'énergie de vibration de HF est transféré à la vibration de la molécule $A B$ de fréquence voisine. Il a peu d'énergie transférée à la translation.

\subsection{Largeurs de raie}

Comme pour les transitions atomiques, les transitions peuvent être élargies par effet Doppler ou par les collisions.

Pour une raie Doppler, on a :

$$
f(v)=\frac{1}{\delta v_{D}} \sqrt{\frac{\ln 2}{\pi}} \exp \left[-\left(\frac{v-v_{0}}{\delta v_{D}}\right)^{2} \ln 2\right] ; \delta v_{D}=\frac{v}{c} \sqrt{\frac{2 R T \ln 2}{M}}
$$

et pour une raie élargie par collisions, on a :

$$
f(v)=\frac{\delta v_{L}}{\pi\left[\left(v-v_{o}\right)^{2}+\left(\delta v_{L}\right)^{2}\right]}
$$

où $\delta v_{D}$ et $\delta v_{L}$ sont les demi-largeurs à mi-hauteur.

Pour une raie mixte, le profil est donné par la fonction de Voigt (Armstrong, 1967). 


\subsection{Coefficients d'amplification}

Pour une transition de la branche $\mathrm{P}$, le coefficient d'amplification s'écrit :

$$
\alpha(v)=\frac{8 \pi^{3}}{3 h c} v(v, J) J\left|\mu_{v+1, v}\right|^{2} f(v)\left(\frac{n_{v}+1, J-1}{g_{v}+1, J-1}-\frac{n_{v,} J}{g_{v}, J}\right)
$$

où $|\mu|^{2}$ est le moment dipolaire de la transition, $n_{v}+1, J-1$ et $n_{v, J}$ les populations des niveaux supérieur et inférieur ; $g_{v}+1, J-1$ et $g_{v} J$ sont les poids statistiques des niveaux et $f(v)$, le facteur de raie.

Pour la plupart des transitions laser, le coefficient d'amplification est compris entre 0,5 et 5 $\%$ par $\mathrm{cm}$.

\section{LASERS $\mathrm{CO}_{2}$}

\subsection{Généralités}

\subsubsection{Niveaux d'énergie}

La figure 1 montre les premiers niveaux vibrationnels de la molécule $\mathrm{CO}_{2}$. Dans l'ordre croissant d'énergie, on trouve les niveaux $01^{1} 0,02^{0} 0+02^{2} 0$ (mode $\left.v_{2}\right), 10^{0} 0\left(\right.$ mode $\left.v_{1}\right)$ et $00^{0} 1$ (mode $v_{3}$ ). Le premier niveau de vibration de l'azote est très proche du niveau $00^{0} 1 \mathrm{du}$ $\mathrm{CO}_{2}$, ce qui permet un transfert d'énergie facile de $\mathrm{N}_{2}$ vers $\mathrm{CO}_{2}$ par échange $\mathrm{V}-\mathrm{V}$. Les deux transitions laser sont $00^{0} 1-10^{0} 0$ vers $10,6 \mu \mathrm{m}$ et $00^{0} 1-02^{\circ} 0$ vers $9,6 \mu \mathrm{m}$.
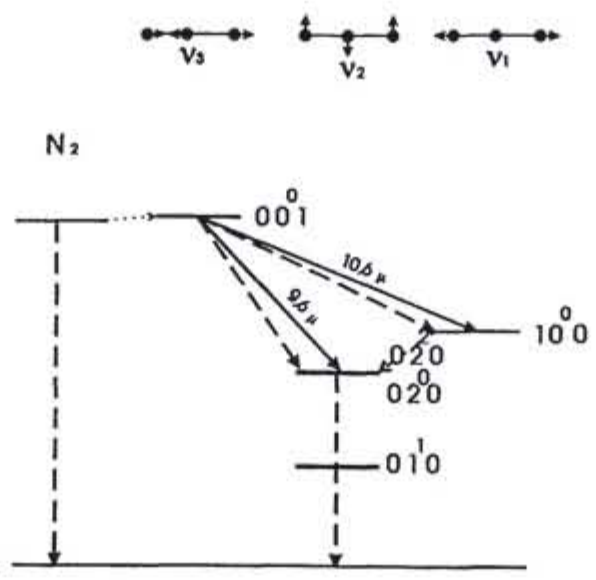

Figure 1: Diagramme des premiers niveaux vibrationnels des molécules $\mathrm{N}_{2}$ et $\mathrm{CO}_{2}$

\subsubsection{Echanges $V$-T et $V$-V}

Le processus V-T essentiel est la désexcitation du niveau $01^{1} 0$ car il contrôle la désexcitation des niveaux inférieurs $10^{\circ} 0$ et $02^{\circ} 0$ des deux transitions laser. Ce processus s'écrit : 


$$
\mathrm{CO}_{2}\left(01^{1} 0\right)+\mathrm{M} \rightarrow \mathrm{CO}_{2}\left(00^{0} 0\right)+\mathrm{M}+\Delta \mathrm{E}=667 \mathrm{~cm}^{-1}
$$

Il est lent pour $\mathrm{M}=\mathrm{CO}_{2}$ et $\mathrm{N}_{2}\left(\mathrm{k} \approx 5.10^{-15} \mathrm{~cm}^{3} / \mathrm{s}\right)$ et relativement rapide pour $\mathrm{M}=\mathrm{He}(\mathrm{k} \approx$ $\left.1,2.10^{-13} \mathrm{~cm}^{3} / \mathrm{s}\right)$. L'addition d'hélium au mélange $\mathrm{CO}_{2}-\mathrm{N}_{2}$ permet donc de réduire le temps de vie du niveau inférieur et de diminuer sa population.

Avec un mélange contenant au moins $50 \%$ d'hélium et aux pressions utilisées dans les lasers $\mathrm{CO}_{2}$, les modes de vibration $\mathrm{v}_{1}$ et $\mathrm{v}_{2}$ sont en équilibre thermodynamique à une température TV $1 \approx T V 2$ proche de la température $T$ du gaz, ce qui veut dire que les populations des niveaux sont déterminées par la distribution de Boltzmann à la température TV1. Toutes les populations des modes $v_{1}$ et $v_{2}$ sont donc fixées par celles du niveau $01^{1} 0$.

Le niveau supérieur $00^{0} 1$ de la transition se désexcite par les processus V-V du type :

$$
\mathrm{CO}_{2}\left(00^{0} 1\right)+\mathrm{M} \rightarrow \mathrm{CO}_{2}(030)+\mathrm{M}
$$

suivis du processus V-T décrit précédemment. Ces processus sont lents $\left(\mathrm{k} \approx 5.10^{-15} \mathrm{~cm}^{3} / \mathrm{s}\right.$.

Le processus V-V le plus important est celui permettant d'exciter le niveau $00^{0} 1$ à partir du premier niveau excité de l'azote :

$$
\mathrm{N}_{2}(\mathrm{v}=1)+\mathrm{CO}_{2}\left(00^{0} 0\right) \rightarrow \mathrm{N}_{2}(\mathrm{v}=0)+\mathrm{CO}_{2}\left(00^{0} 1\right)+\Delta \mathrm{E}=18 \mathrm{~cm}^{-1}
$$

Ce processus est très rapide $\left(k \approx 6.10^{-13} \mathrm{~cm}^{3} / \mathrm{s}\right)$.

Différents travaux expérimentaux et théoriques ont montré que les populations des niveaux du mode $v_{3}$ se répartissaient suivant une loi de Boltzmann à la température TV3 fixée par la population du niveau $00^{0} 1$.

\subsubsection{Equations de relaxation des populations}

Basov a établi les équations de relaxation des énergies $E_{1}, E_{2}$ et $E_{3}$ des modes $v_{1}, v_{2}$ et $v_{3}$ en supposant les trois modes en équilibre thermodynamique aux températures respectives TV1, TV2 et TV3.

La résolution de ces équations assez complexes permet de calculer ensuite sans difficulté les différentes populations vibrationnelles.

\subsubsection{Coefficient d'amplification}

Il s'obtient facilement à partir de l'expression 1 . Typiquement pour TV3 $=2000 \mathrm{~K}$ et TV1 $=$ TV2 $=\mathrm{T}=300 \mathrm{~K}$, sa valeur est d'environ $1 \%$ par $\mathrm{cm}$ pour un mélange $\mathrm{CO}_{2}-\mathrm{N}_{2}-\mathrm{He}$ contenant $5 \%$ de $\mathrm{CO}_{2}$.

\subsection{Excitation par décharges électriques}

Dans une décharge électrique, continue ou impulsionnelle, les niveaux $\mathrm{N}_{2}(\mathrm{v})$ et $\mathrm{CO}_{2}\left(\mathrm{v}_{1}, \mathrm{v}_{2}\right.$ et $v_{3}$ ) sont peuplés par des collisions électroniques du type :

$$
\begin{gathered}
\mathrm{CO}_{2}(000)+\mathrm{e} \rightarrow \mathrm{CO}_{2}(001)+\mathrm{e}^{\prime} \\
\mathrm{CO}_{2}(000)+\mathrm{e} \rightarrow \mathrm{CO}_{2}(010)+\mathrm{e}^{\prime} \\
\mathrm{N}_{2}(0)+\mathrm{e} \rightarrow \mathrm{N}_{2}(\mathrm{v})+\mathrm{e}^{\prime}
\end{gathered}
$$

L'efficacité de la collision dépend de la section efficace et de la fonction de distribution des électrons. Cette dernière, pour un mélange de gaz donné, est seulement fonction du rapport $\mathrm{E} / \mathrm{N}$ 
où E est le champ électrique appliqué et $\mathrm{N}$ la densité de particules neutres. En fait, les plasmas mis en jeu étant très peu ionisés ( $\mathrm{ne} / \mathrm{N}=10^{-5}$ ), la densité des neutres est celle du gaz.

\subsubsection{Fonction de distribution des électrons}

La répartition énergétique des électrons est régie par l'équation de Boltzmann dont la forme simplifiée est :

$$
\frac{1}{3}\left(\frac{E}{N}\right)^{2} \frac{u}{Q_{m}} \frac{d f}{d u}=-\sum_{j} \int_{u}^{u+u_{j}} u Q_{j}(u) f(u) d u
$$

Dans cette équation, u est l'énergie de l'électron, $u_{j}$ est la perte d'énergie et $Q_{j}(u)$ la section efficace inélastique du processus $\mathrm{j}$. $\mathrm{Q}_{\mathrm{m}}$ est la section de transfert de quantité de mouvement et $\mathrm{E} / \mathrm{N}$ le champ électrique réduit.

$$
\text { La fonction } f(u) \text { est normalisée } \int_{0}^{-} \sqrt{u} f(u) d u=1 \text {. }
$$

La fonction $\mathrm{f}(\mathrm{u})$ rassemble toutes les informations utiles sur le plasma. Elle permet de connaître l'énergie moyenne des électrons $\bar{u}_{\mathrm{r}}$, leur vitesse d'entraînement $\mathrm{v}_{\mathrm{d}}$, le coefficient de diffusion De et les différents cœfficients d'excitation, d'ionisation et d'attachement. Nighan a présenté, en 1970 , les premiers résultats relatifs aux mélanges $\mathrm{CO}_{2}-\mathrm{N}_{2}-\mathrm{He}$. Parmi d'autres résultats ultérieurs, citons ceux de Lowke, Phelps et Irwin (1973) et ceux concernant la modélisation du laser $\mathrm{CO}_{2}$, de Bullis (1972) et de Mills (1974).

\subsubsection{Populations vibrationnelles - Coefficient d' excitation}

L'excitation des niveaux vibrationnels est déterminée par le produit $\mathrm{k}_{\mathrm{j}} \mathrm{n}_{\mathrm{e}} \mathrm{N}$ où $\mathrm{k}_{\mathrm{j}}$ est le coefficient d'excitation calculé d'après $\mathrm{f}(\mathrm{u}), \mathrm{n}_{\mathrm{e}}$ la densité électronique du plasma et $\mathrm{N}$ la densité du gaz considéré $\left(\mathrm{N}_{2}\right.$ ou $\left.\mathrm{CO}_{2}\right)$. La densité électronique $\mathrm{n}_{\mathrm{e}}$ peut se calculer par une équation annexe ou se déduire du courant de décharge mesuré par la relation $\mathrm{j}=\mathrm{n}_{\mathrm{e}} \mathrm{ev}$.

$L$ 'introduction des termes d'excitation $\mathrm{k}_{\mathrm{j}} \mathrm{n}_{\mathrm{e}} \mathrm{N}$ dans les équations de relaxation décrites au $\S$ 1.3 permet de calculer les populations vibrationnelles du $\mathrm{CO}_{2}$ et les coefficients d'amplification. L'intensité laser sur la transition rotationnelle de plus grand gain se calcule en prenant en compte les caractéristiques optiques de la cavité.

\subsection{Lasers $\mathrm{CO}_{2}$ continus}

\subsubsection{Lasers $\mathrm{CO}_{2}$ industriels}

3.3.1.1 Lasers à décharge longitudinale. Les lasers $\mathrm{CO}_{2}$ de puissance utilisent le plus souvent des décharges électriques longitudinales où l'axe optique, le champ électrique et la direction de l'écoulement sont confondus. Sur les réalisations récentes, les décharges utilisées sont du type $\mathrm{RF}$ et le champ électrique est alors appliqué transversalement (voir figure 2).

Typiquement, la pression totale régnant dans les tubes à décharge est de l'ordre de 100 mbars, la vitesse du mélange gazeux d'environ $100-150 \mathrm{~m} / \mathrm{s}$ et le diamètre des tubes varie de 1 à $3,5 \mathrm{~cm}$. La puissance spécifique de la décharge varie de 5 à $20 \mathrm{~W} / \mathrm{cm}^{3}$ selon le type de décharge mis en jeu. Le rendement, exprimé comme le rapport de la puissance laser émise à la puissance fournie à la décharge, varie de 15 à $25 \%$. Les figures 3 et 4 montrent les caractéristiques calculées (Brunet,1982) d'un tube à décharge de $30 \mathrm{~cm}$ de longueur.

Le principal avantage des lasers à décharge longitudinale est l'excellent remplissage du volume actif qu'il permet d'obtenir. Le volume délimité par les modes optiques d'ordre peu élevé (TE 00 et TE 01 ) est voisin du volume de décharge. Il s'ensuit un bon rendement dépassant souvent $20 \%$. 


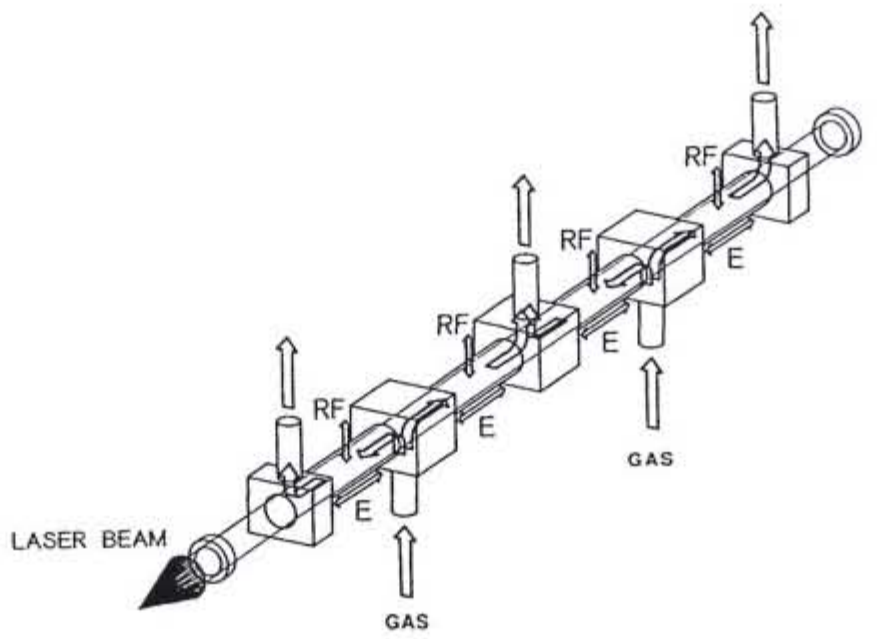

Figure 2: Configuration géométrique d'un laser $\mathrm{CO}_{2}$ à décharge longitudinale

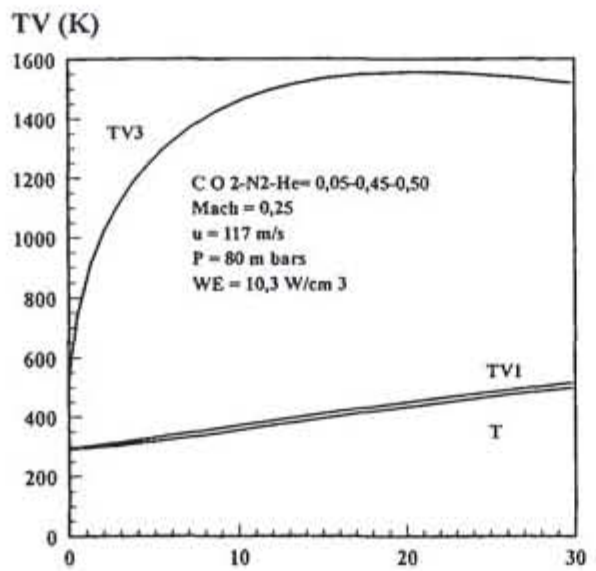

Figure 3 : Températures vibrationnelles suivant le sens de l'écoulement

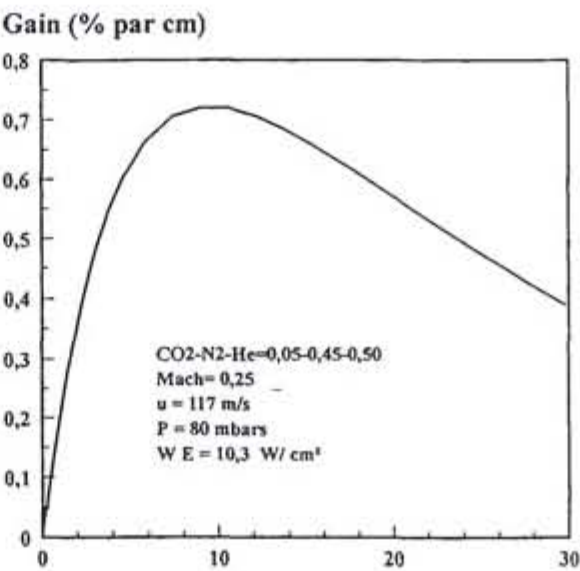

Figure 4 :Coefficient d'amplification suivant le sens de l'écoulement

Le principal inconvénient résulte des pertes de charge élevées de la boucle de circulation de gaz. Cela impose d'utiliser un ventilateur présentant un taux de compression élevé $(>1,4)$.

Pour des raisons fondamentales liées, entre autres, au temps de relaxation du niveau $00^{0} 1$, la longueur des tubes à décharge doit être limitée à 20-30 cm. De ce fait, les lasers délivrant plusieurs kW comportent de 6 à 16 tubes placés optiquement en série et aérodynamiquement en parallèle. Différentes dispositions ont été étudiées et sont exploitées commercialement (en alignement : Adron, Rofin Sinar, PCR, .... en carré : Trumpf, Salvo, 1992, en triangle : Wegmann-Baasel, Wegmann, 1992). 
3.3.1.2 Lasers à décharge transversale. Pour les très fortes puissances ( $>10 \mathrm{~kW})$, la technique de décharge longitudinale doit être abandonnée car elle ne permet pas de refroidir rapidement un grand volume de gaz. On utilise alors une géométrie à décharge transversale où la direction de l'écoulement est perpendiculaire à l'axe optique (voir figure 5). Le temps de séjour des molécules dans la décharge est court et l'échauffement du gaz est réduit.

\section{PERPENDICULAR AXES}

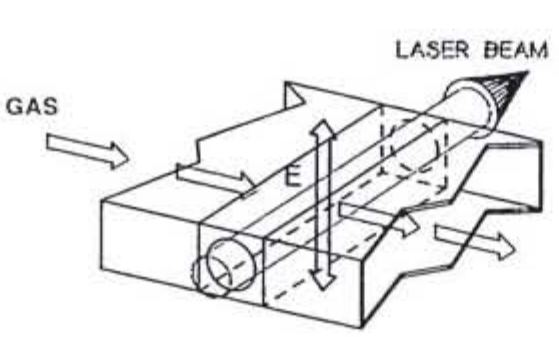

\section{PERPENDICULAR AXES}

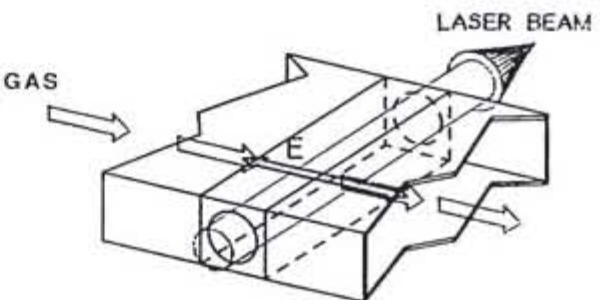

Figure 5 : Configuration géométrique d'un laser à décharge transversale

Le principal inconvénient de la géométrie à écoulement transversal est la nécessité d'utiliser une cavité optique instable pour obtenir un faisceau laser de bonne qualité optique.

Le principal avantage est la possibilité d'utiliser un ventilateur à faible taux de compression car les pertes de charge de la boucle de circulation de gaz sont faibles. Des lasers de plus de 10 $\mathrm{kW}$ sont commercialisés par UTRC et Rofin-Sinar.

\subsubsection{Lasers $\mathrm{CO}_{2}$ de très forte puissance}

Au début des années 1970 , des lasers $\mathrm{CO}_{2}$ de très forte puissance (>100 kW) ont été réalisés par d'autres techniques (à excitation électrique ou thermique, type GDL).

\subsection{Laser $\mathrm{CO}_{2}$ guide d'onde}

Pour les petites puissances continues ( 5 à $20 \mathrm{~W}$ ), il est possible de réaliser des lasers $\mathrm{CO}_{2}$ particulièrement compacts en utilisant la technologie guide d'onde. La décharge est établie dans un tube de faible section (ronde ou carrée $<10 \mathrm{~mm}^{2}$ ) et typiquement de $20-50 \mathrm{~cm}$ de longueur. Le tube est réalisé dans un matériau isolant bon conducteur de la chaleur $\left(\mathrm{Al}_{2} \mathrm{O}_{3}, \mathrm{BeO}, \ldots\right)$ qui permet de refroidir le mélange gazeux par conduction vers les parois et non par circulation, le tube étant scellé. Les réalisations récentes utilisent une décharge transversale à couplage capacitif (absence d'électrodes dans le gaz), la fréquence d'excitation étant de l'ordre de $100 \mathrm{MHz}$. Le mélange utilisé est généralement $\mathrm{CO}_{2}-\mathrm{N}_{2}-\mathrm{He}=0,15-0,15-0,70$ à $\mathrm{P} \approx 100$ torrs, avec quelquefois l'addition d'un peu de xénon.

$\mathrm{La}$ durée de vie d'un laser $\mathrm{CO}_{2}$ guide d'onde est d'environ $5000 \mathrm{~h}$. 


\subsection{Laser $\mathrm{CO}_{2}$ impulsionnels}

En excitant l'azote et le $\mathrm{CO}_{2}$ dans une décharge impulsionnelle à haute pression, il est possible d'obtenir une émission laser de très grande puissance crête. La difficulté essentielle est de réaliser une décharge stable et uniforme dans un mélange $\mathrm{CO}_{2}-\mathrm{N}_{2}-\mathrm{He}$ à haute pression. Comme le fonctionnement de base n'est pas différent de celui d'un laser $\mathrm{CO}_{2}$ continu, il faut conserver le même rapport $\mathrm{E} / \mathrm{N}\left(\sim 4 \cdot 10^{-16} \mathrm{~V} \cdot \mathrm{cm}^{2}\right)$, ce qui conduit, si la pression est voisine de 1 bar, à des champs électriques d'environ $10 \mathrm{kV} / \mathrm{cm}$. Pour des raisons pratiques, on est donc conduit à utiliser une décharge transversale.

Pour obtenir une décharge uniforme, il est nécessaire de préioniser le milieu gazeux, en général juste avant l'application de l'impulsion de tension. Les électrons de préionisation (ne = $105 / \mathrm{cm}^{3}$ ) sont ensuite multipliés très rapidement par le champ électrique appliqué et produisent un courant de décharge intense.

Deux techniques de préionisation sont possibles : l'une utilise un faisceau d'électrons de grande énergie et l'autre l'effet corona.

Les premiers lasers $\mathrm{CO}_{2}$ TEA (transversely excited atmospheric pressure) développés ont utilisé une préionisation par effet corona quoique le mécanisme de préionisation ait été mal compris à l'époque. La figure 6 montre une vue schématique du dispositif de décharge mis au point par Dumanchin et Rocca Serra en 1970.

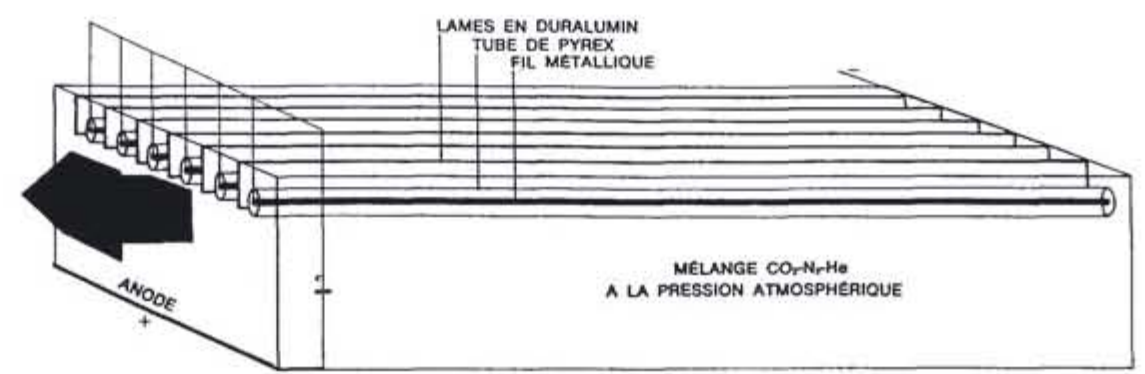

Figure 6 : Vue schématique de la décharge transversale à préionisation corona

L'effet corona prend naissance à la surface du tube de Pyrex, sous l'influence du champ électrique intense produit entre le fil et la lame métallique. Les électrons ainsi créés ont le temps de remplir tout l'espace interélectrode avant que la tension appliquée atteigne son maximum. On obtient ainsi une décharge uniforme dans de grands volumes. Le schéma électrique du circuit est donné par la figure 7. Typiquement, un laser TEA de ce type peut délivrer une énergie spécifique d'environ $40 \mathrm{~J} / \mathrm{h}$ avec un rendement de $16 \%$.

L'impulsion laser d'une durée totale de $1 \mu$ s présente un pic de courte durée $(60 \mathrm{~ns})$ contenant la moitié de l'énergie. Le coefficient d'amplification atteint $4,6 \%$ par cm.

Le plus gros laser réalisé à l'époque a délivré une énergie de $130 \mathrm{~J}$ par impulsion.

La préionisation par faisceau d'électrons a permis, quelques années plus tard, d'exciter de très grands volumes au prix d'une technologie onéreuse et délicate. Le laser "Big Bang" réalisé par Avco Everett (USA) en 1974 a délivré une énergie de $2 \mathrm{~kJ}$ par impulsion à partir d'un volume de décharge de 401 . 


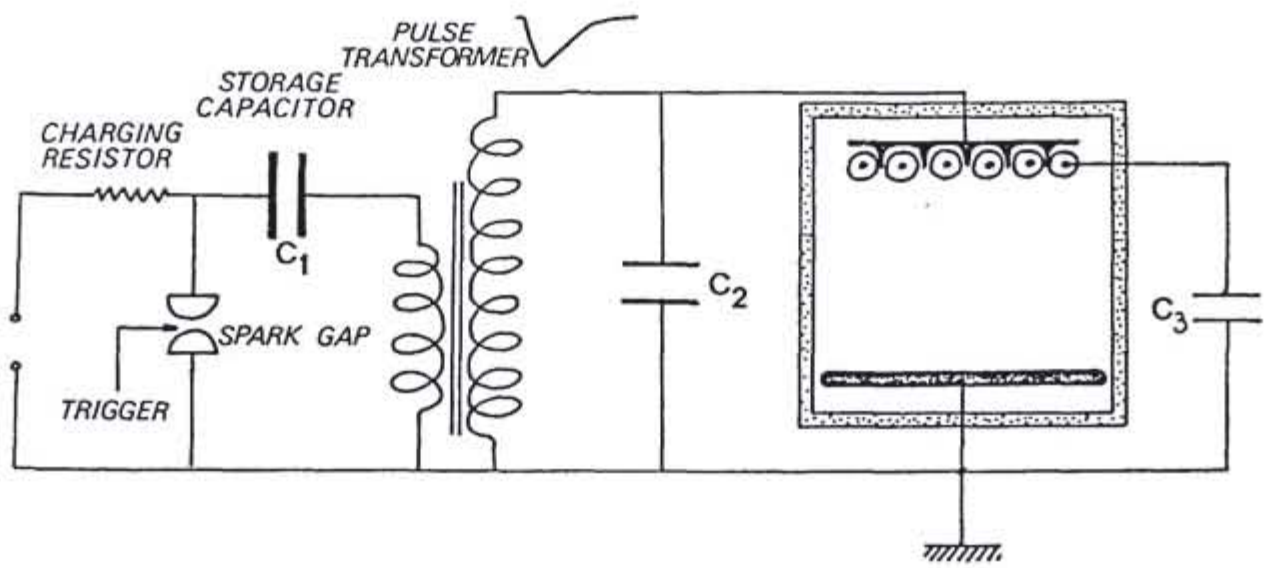

Figure 7 : Schéma du circuit électrique du laser $\mathrm{CO}_{2}$ TEA

\section{LASERS CHIMIQUES}

\subsection{Généralités}

Dans un laser chimique, les molécules excitées sont produites par une réaction du type :

$$
\mathrm{A}+\mathrm{BC} \rightarrow \mathrm{AB}(\mathrm{v})+\mathrm{C}
$$

L'émission laser est obtenue sur les transitions de vibration-rotation de la molécule $\mathrm{AB}$ :

$$
\mathrm{AB}(\mathrm{v}) \Subset \mathrm{AB}(\mathrm{v}-1)+\mathrm{hn}
$$

Dans la réaction de base, la plus grande partie de l'énergie $(\Delta \mathrm{H})$ de la réaction apparaît initialement sous forme vibrationnelle et n'est transformée en chaleur que plus tard par les mécanismes de relaxation $\mathrm{V}$-T.

\subsection{Processus élémentaires}

\subsubsection{Réactions de production}

Il existe relativement peu de réactions donnant lieu à l'excitation vibrationnelle d'un des produits de réaction. Les principaux systèmes connus sont indiqués dans le tableau ci-après.

Les vitesses de réactions ont été mesurées pour quelques systèmes. Pour la réaction la plus utilisée $\mathrm{F}+\mathrm{H}_{2}$, on a :

$$
\begin{aligned}
& \mathrm{F}+\mathrm{H}_{2}(0) \rightarrow \mathrm{HF}(\mathrm{v}=1)+\mathrm{H} ; \mathrm{k}=5 \cdot 10^{-12} \mathrm{~cm}^{3} / \mathrm{s} \\
& \mathrm{F}+\mathrm{H}_{2}(0) \rightarrow \mathrm{HF}(\mathrm{v}=2)+\mathrm{H} ; \mathrm{k}=1,6.10^{-11} \mathrm{~cm}^{3} / \mathrm{s} \\
& \mathrm{F}+\mathrm{H}_{2}(0) \rightarrow \mathrm{HF}(\mathrm{v}=3)+\mathrm{H} ; \mathrm{k}=7,7.10^{-12} \mathrm{~cm}^{3} / \mathrm{s}
\end{aligned}
$$




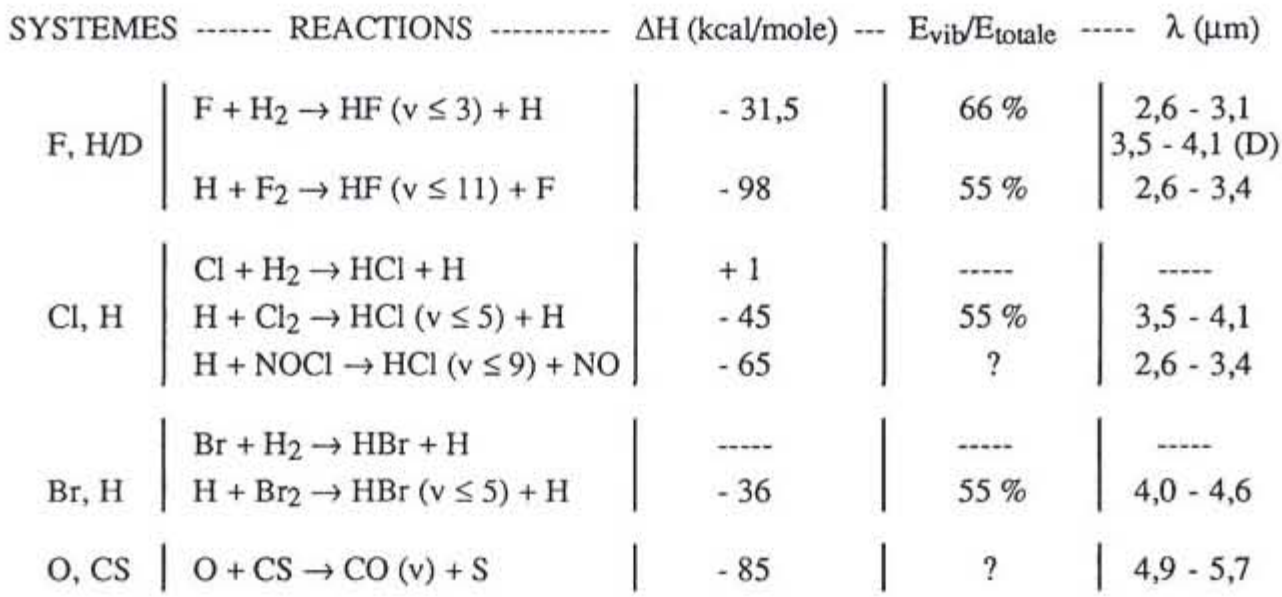

\subsubsection{Processus collisionnels - Echanges $V$-T et $V$-V}

Le caractère très polaire de la molécule $\mathrm{HF}$ fait que tous les processus collisionnels sont plus rapides que ceux mis en jeu dans les mélanges $\mathrm{CO}_{2}-\mathrm{N}_{2}-\mathrm{He}$; ils sont mal connus pour les niveaux vibrationnels élevés. A titre d'exemple, on a mesuré :

- échanges V-T

$$
\begin{aligned}
& \mathrm{HF}(1)+\mathrm{HF}(0) \rightarrow 2 \mathrm{HF}(0) ; \mathrm{k}=1,7.10^{-12} \mathrm{~cm}^{3} / \mathrm{s} \\
& \mathrm{HF}(2)+\mathrm{HF}(0) \rightarrow \mathrm{HF}(1)+\mathrm{HF}(0) ; \mathrm{k}=4,6.10^{-12} \mathrm{~cm}^{3} / \mathrm{s}
\end{aligned}
$$

\section{- échanges $\mathrm{V}-\mathrm{V}$}

$$
\begin{aligned}
& \mathrm{HF}(2)+\mathrm{HF}(0) \rightarrow \mathrm{HF}(1)+\mathrm{HF}(1) ; \mathrm{k}=6,4 \cdot 10^{-12} \mathrm{~cm}^{3} / \mathrm{s} \\
& \mathrm{HF}(3)+\mathrm{HF}(0) \rightarrow \mathrm{HF}(2)+\mathrm{HF}(1) ; \mathrm{k}=1,2 \cdot 10^{-11} \mathrm{~cm}^{3} / \mathrm{s}
\end{aligned}
$$

Les coefficients de désexcitation sont du même ordre de grandeur que les coefficients de production, ce qui veut dire que la réalisation d'un laser chimique HF/DF n'est pas facile. Une compilation des différents coefficients est présentée dans l'ouvrage de Gross et Bott (1976). On y trouvera aussi les différents aspects de la modélisation d'un laser chimique HF, aspects similaires à ceux présentés pour le laser $\mathrm{CO}_{2}$.

\subsection{Techniques d'initiation}

La principale difficulté pour réaliser un laser chimique HF est de produire les atomes $\mathrm{F}$ de base. Plusieurs techniques ont été utilisées pour produire ces atomes à partir d'un composé fluoré :
1 - la photodissociation
2 - la dissociation thermique
3 - la dissociation électrique
4 - la dissociation par faisceau d'électrons rapides
5 - l'utilisation d'une réaction annexe

\subsection{Lasers chimiques HF/DF continus de grande puissance}

Ils utilisent la dissociation thermique du fluor $\mathrm{F}_{2}$ ou d'un composé fluoré moins dangereux, $\mathrm{NF}_{3}$. On réalise dans une chambre appropriée, la combustion de $\mathrm{F}_{2}$ (en excès par rapport à la 
composition stoïchiométrique) et de l'hydrogène (ou $\left.\mathrm{C}_{2} \mathrm{H}_{4}\right)$ en présence d'un gaz diluant, l'hélium qui sert à abaisser la température du mélange gazeux vers $2000 \mathrm{~K}$. On obtient ainsi un mélange à 3 bars de composition suivante :

$$
\mathrm{F}-\mathrm{HF}-\mathrm{He} \approx 0,10-0,15-0,75
$$

Cette source d'atomes F n'est pas utilisable directement car la température et la pression sont beaucoup trop élevées. On fait alors subir au mélange une détente très rapide (pour éviter la recombinaison des atomes $\mathrm{F}$ en $\mathrm{F}_{2}$ ) à travers de petites tuyères qui abaisse simultanément la température et la pression. On dispose ainsi à la sortie des tuyères d'un mélange froid ( $\mathrm{T}<$ $300 \mathrm{~K}$ ) et à basse pression ( $\mathrm{P}<10$ torrs). Pour réaliser la réaction $\mathrm{F}+\mathrm{D}_{2} \rightarrow \mathrm{DF}(\mathrm{v})+\mathrm{F}$, on intercale entre les tuyères ci-dessus d'autres tuyères alimentées en mélange $D_{2}+\mathrm{He}$; leurs jets interagissent avec les précédents pour créer des zones de mélange où les atomes F réagissent avec les molécules $\mathrm{D}_{2}$.

Typiquement, les tuyères ont un col de $0,1 \mathrm{~mm}$ et une largeur de sortie de 3 à $4 \mathrm{~mm}$. Leur hauteur peut varier de 2 à $20 \mathrm{~cm}$ suivant la puissance laser.

La figure 8 montre une vue schématique d'un laser DF d'environ $10 \mathrm{~kW}$ réalisé vers 1980 aux Laboratoires de Marcoussis.

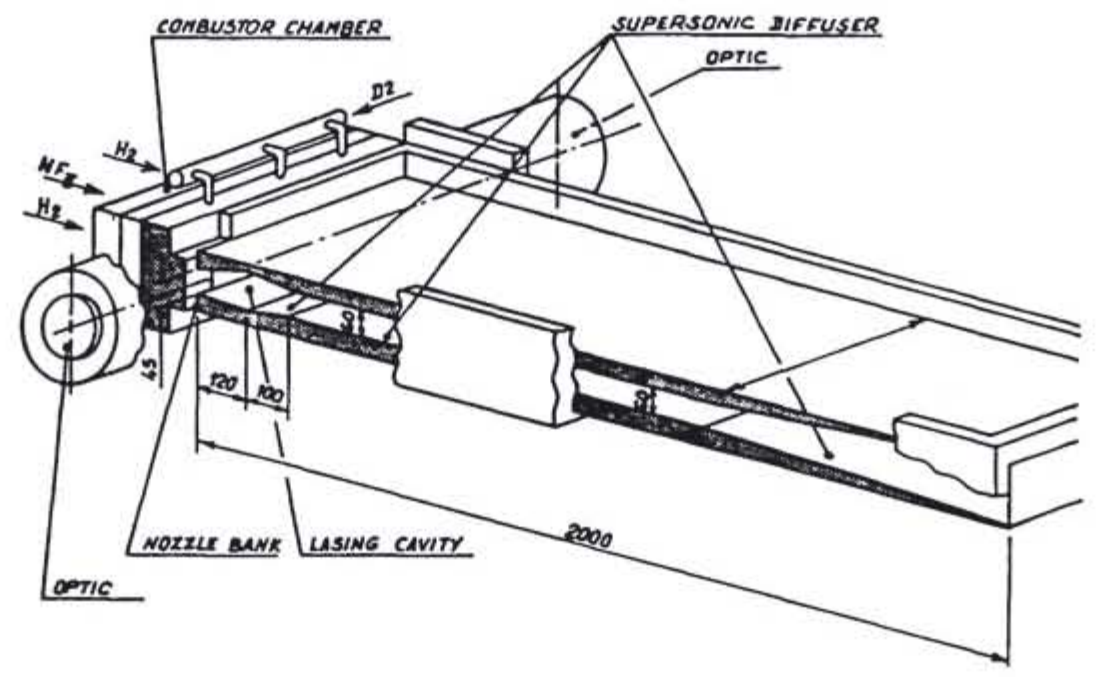

Figure 8 : Vue schématique d'un laser chimique DF à combustion

D'autres détails peuvent être trouvés dans le livre de Gross et Bott déjà cité et dans l'article de Wilson (1980).

Le plus puissant laser chimique DF continu a été réalisé par TRW en 1980 : c'est le laser MIRACL (Mid-Infrared Advanced Chemical Laser) construit pour la Navy. La puissance délivrée dans la plage $3,6-4,0 \mu \mathrm{m}$ est de $2 \mathrm{MW}$. Un laser chimique HF tout aussi puissant mais de conception différente car destiné à fonctionner dans l'espace est en cours d'expérimentation : c'est le laser ALPHA réalisé aux USA pour le compte de la SDIO (Strategic Defense Initiative Organization). 


\subsection{Lasers chimiques HF/DF impulsionnels}

Deux catégories de lasers HF/DF impulsionnels ont été étudiées : l'une utilise la réaction $\mathrm{F}+\mathrm{H}_{2}$ et l'autre la réaction en chaîne $\mathrm{F}_{2}+\mathrm{H}_{2}$, réaction beaucoup plus difficile à maîtriser.

\subsubsection{Laser HFIDF à excitation électrique}

La production des molécules excitées est obtenue dans une décharge électrique assez semblable à celle décrite pour les lasers $\mathrm{CO}_{2} \mathrm{TEA}$. Cependant, les propriétés électroniques particulières du mélange gazeux utilisé $\left(\mathrm{SF}_{6}-\mathrm{Ne}-\mathrm{H}_{2}\right)$ imposent des contraintes sévères au circuit électrique de commutation. C'est la raison pour laquelle, à LASERDOT, nous avons choisi d'utiliser la technique du photodéclenchement (Lacour 1987, Brunet 1990).

La production des atomes $\mathrm{F}$ résulte de la dissociation électrique de $\mathrm{SF}_{6}$ suivant les processus :

$$
\begin{gathered}
\mathrm{SF}_{6}+\mathrm{e} \rightarrow \mathrm{SF}_{5}+\mathrm{F}+2 \mathrm{e} \\
\mathrm{SF}_{6}+\mathrm{e} \rightarrow \mathrm{SF}_{5}+\mathrm{F} \\
\mathrm{SF}_{6}+\mathrm{e} \rightarrow \mathrm{SF}_{6}+\mathrm{e} \rightarrow \mathrm{SF}_{5}+\mathrm{F}+\mathrm{e}
\end{gathered}
$$

Dans la maquette laser réalisée, la décharge d'un volume de $0,51\left(3 \times 4 \times 45 \mathrm{~cm}^{3}\right)$ est préionisée par effet corona. La pression du mélange $\mathrm{SF}_{6}-\mathrm{Ne}-\mathrm{C}_{2} \mathrm{H}_{6}=0,50-0,45-0,05$ est de 0,2 bar et la tension maximale de décharge de $42 \mathrm{kV}$. Dans ces conditions, l'énergie laser par impulsion atteint $5,5 \mathrm{~J}$; la durée d'impulsion est de $160 \mathrm{~ns}$.

Une puissance moyenne élevée peut être obtenue en fonctionnement répétitif. Pour cela, il faut renouveler complètement le mélange gazeux dans l'espace interélectrode entre deux impulsions. Un dispositif de circulation de gaz, comme celui figuré sur la figure 9, permet d'atteindre cet objectif (Brunet et al., 1993).

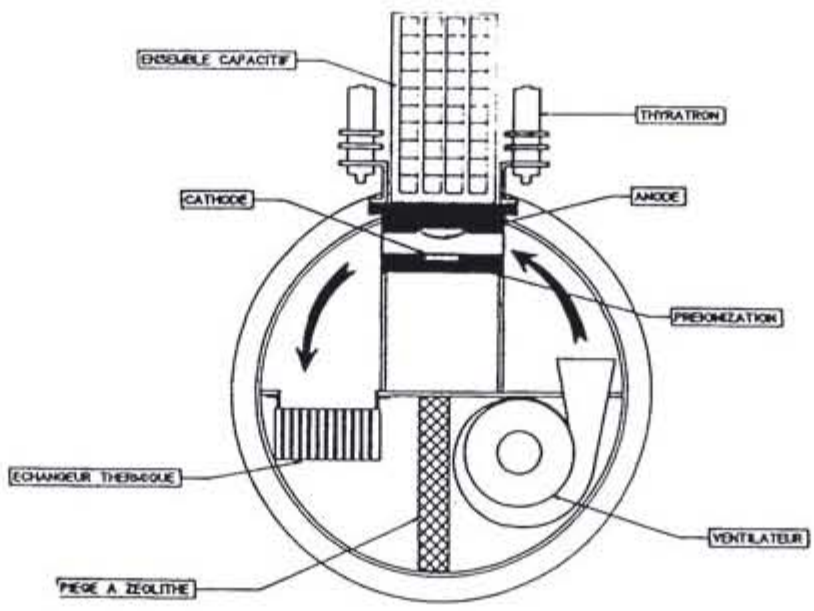

Figure 9: Coupe schématique du laser chimique HF/DF pulsé répétitif

Dans des conditions expérimentales sensiblement analogues aux précédentes, la puissance laser moyenne délivrée par le système a atteint $500 \mathrm{~W}$ à une cadence de $110 \mathrm{~Hz}$.

La figure 10 montre des impulsions laser observées à une cadence de $110 \mathrm{~Hz}$. 


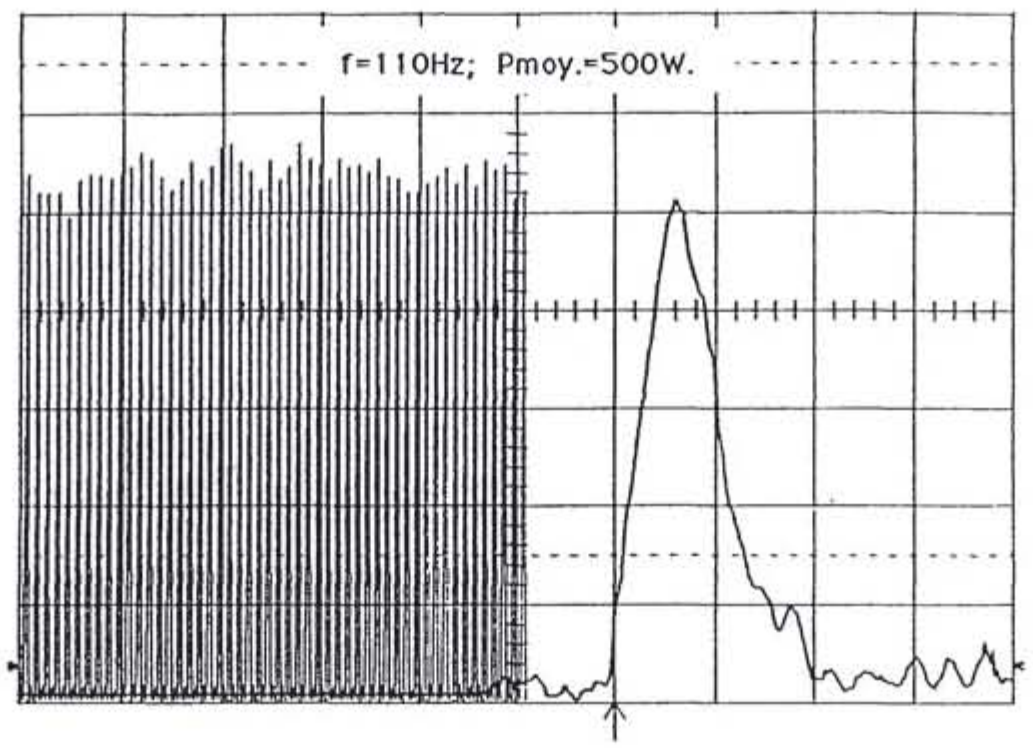

Figure 10 : Séquence de 51 impulsions consécutives à $110 \mathrm{~Hz}$ Vue agrandic de la $51^{\mathrm{ème}}$ impulsion (200 ns/div)

\subsubsection{Laser HF/DF à réaction en chaîne}

La réaction mise en jeu $\mathrm{F}_{2}+\mathrm{H}_{2}$ se déroule en deux étapes élémentaires :

$$
\begin{aligned}
& \mathrm{F}+\mathrm{H}_{2} \rightarrow \mathrm{HF}(\mathrm{v} \leq 3)+\mathrm{H} ; \Delta \mathrm{H}=-33 \mathrm{kcal} / \mathrm{mole} \\
& \mathrm{H}+\mathrm{F}_{2} \rightarrow \mathrm{HF}(\mathrm{v} \leq 11)+\mathrm{F} ; \Delta \mathrm{H}=-98 \mathrm{kcal} / \mathrm{mole}
\end{aligned}
$$

Les réactions sont difficiles à utiliser du point de vue laser car la deuxième réaction produit des molécules excitées dans des états vibrationnels très élevés qui se désexcitent extrêmement rapidement. La réaction globale doit être ralentie par l'addition d'oxygène pour éviter une explosion prématurée. L'intérêt de ce type de laser est qu'il ne nécessite pratiquement pas d'énergie électrique, une très faible quantité d'atomes F suffisant pour démarrer la réaction.

On a pu montrer que l'énergie laser spécifique était proportionnelle à la pression $\left(F_{2}\right) \sqrt{F / F_{2}}$. Typiquement, pour $\mathrm{P}\left(\mathrm{F}_{2}\right)=100$ torrs et $\mathrm{F} / \mathrm{F}_{2}=0,03$, l'énergie spécifique atteint $30 \mathrm{~J} / 1$.

Les plus gros lasers réalisés délivrent, au coup par coup, une énergie de $5 \mathrm{~kJ}$ par impulsion (Gerber et Patterson, 1974 - Inagaki et al., 1986). Une technologie récente peut permettre d'obtenir des impulsions longues avec un rendement avoisinant $100 \%$ (Dreiling, 1991).

\section{Références}

[1] Armstrong B., J.Q.R.S.T. 7 (1967) pp. 61-88

[2] Basov N., Sov. Phys. Techn. Phys., 13 (1969), 1630-1636.

[3] Brunet H., La Recherche, 32 (1973) 243-252. 
[4] Brunet H. et al., "Pulsed HF chemical using a VUV phototriggered discharge", Eighth

[5] Symposium on Gas Flow and Chemical Lasers, Madrid, 10-14 September 1990, Orza J.M. and Domingo C. (SPIE vol. 1397) pp. 273-276.

[6] Brunet $\mathrm{H}$. et al., "High average power $\mathrm{XeCl}$ and pulsed $\mathrm{HF}$ chemical lasers", AIAA 24th Plasmadynamics and Laser Conference, Orlando, FL, 6-9 July 1993, paper $93-$ 3165.

[7] Bullis R., AIAA Journal, 10 (1972), 407-413.

[8] Dreiling T., "High efficiency long pulse length DF-CO $\mathrm{CO}_{2}$ and DF pulsed chemical lasers", CLEO/IQEC 22 may 1990, paper CTUE2.

[9] Dumanchin R. and Rocca Serra J., "High power density pulsed molecular laser", Sith Q.E.C., Kyoto, Japan, September 1970, pp 18-6.

[10] Gerber R. and Patterson E., J. Appl. Phys. 47 (1976), 3524-3529.

[11] Gross R. and Borr J., Handbook of chemical lasers (John Wiley, New York, 1976).

[12] Herzberg G., Molecular Spectra and Molecular Structure, I. Spectra of diatomic molecules (D. Van Nostrand Company, Inc., New York, 1961), pp. 124-125.

[13] Inagaki H. et al., J. Appl. Phys. 59 (1986), 324-326.

[14] Lacour B. and Vannier C., J. Appl. Phys. 62 (1987), 754-758.

[15] Lowke J., Phelps A. and Irwin B., J. Appl. Phys. 44 (1973) 4664-4671.

16] Mills C., J. Appl. Physics, 45 (1974) 1336-1341.

[17] Nighan W.L., Phys. Rev. A , 2 (1970) 1989-2000.

[18] Salvo P., Industrial Laser Review May (1992) 9-12.

[19] Wegman H., Industrial Laser Review May (1992) 5-8.

[20] Wilson L., J. Phys. France, Colloque C9, supplément au n 11, 41 (1980) pp. 1-8 